International Mathematical Forum, Vol. 9, 2014, no. 24, 1161 - 1176

HIKARI Ltd, www.m-hikari.com

http://dx.doi.org/10.12988/imf.2014.46116

\title{
On Symmetric Nonnegative Matrices with Prescribed Spectrum ${ }^{1}$
}

\author{
Ricardo L. Soto ${ }^{2}$ and Elvis Valero \\ Departamento de Matemáticas \\ Universidad Católica del Norte \\ Antofagasta, Chile
}

Copyright (c) 2014 Ricardo L. Soto and Elvis Valero. This is an open access article distributed under the Creative Commons Attribution License, which permits unrestricted use, distribution, and reproduction in any medium, provided the original work is properly cited.

\begin{abstract}
In this paper we give a sufficient condition for the existence and construction of a symmetric nonnegative matrix with prescribed spectrum, and a sufficient conditon for the existence and construction of a $4 \times 4$ symmetric nonnegative matrix with prescribed spectrum and diagonal entries. This last condition is independent of the sufficient condition given by Fiedler [LAA 9 (1974) 119-142]. We also give some partial answers on an open question of Guo [LAA 266 (1997) 261-270] about symmetric nonnegative matrices.
\end{abstract}

Mathematis Subject Classification: 15A18, 15A51

Keywords: symmetric nonnegative inverse eigenvalue problem

\section{Introduction}

The nonnegative inverse eigenvalue problem (NIEP) is the problem of finding necessary and sufficient conditions for the existence of an entrywise nonnegative matrix with prescribed complex spectrum $\Lambda=\left\{\lambda_{1}, \lambda_{2}, \ldots, \lambda_{n}\right\}$. A complete solution to this problem is only known for lists of $n \leq 4$ numbers. If there

\footnotetext{
${ }^{1}$ Work supported by Fondecyt 1120180, Conicyt Res. 2093, Chile

${ }^{2}$ Corresponding author
} 
exists a nonnegative matrix $A$ with spectrum $\Lambda$, we say that $\Lambda$ is realizable and that $A$ is the realizing matrix. If the nonnegative matrix $A$ is required to be symmetric we have the symmetric nonnegative inverse eigenvalue problem (SNIEP). The first results related with the SNIEP were obtained by Fiedler [2] in 1974. Sufficient conditions for the problem to have a solution have been obtained, among other, in chronological order, in [2, 11, 8, 9, 5]. In [12], Spector gives a necessary and sufficient condition for the SNIEP, in the case $n=5$ with $\sum_{i=1}^{5} \lambda_{i}=0$ :

Theorem 1.1 Spector [12] Let $\Lambda=\left\{\lambda_{1}, \lambda_{2}, \ldots, \lambda_{5}\right\}$ be a list of real numbers with $\sum_{i=1}^{5} \lambda_{i}=0$. Then $\Lambda$ is the spectrum of a symmetric nonnegative matrix if and only if

i) $S_{1}(\Lambda)=\sum_{i=1}^{5} \lambda_{i}=0$

ii) $S_{3}(\Lambda)=\sum_{i=1}^{\overline{5}} \lambda_{i}^{3} \geq 0$,

iii) $\lambda_{2}+\lambda_{5} \leq 0$.

Based in a result of Horn [4] we give, in section 2, a new constructible sufficient condition for the existence of a symmetric nonnegative matrix with prescribed spectrum.

The following result, which has been exploited with success in connection with the NIEP, is a rank-r perturbation result, due to Rado and introduced by Perfect [6], which shows how to modify $r$ eigenvalues of an $n \times n$ matrix, via a $r a n k-r$ perturbation, without changing any of the remaining $n-r$ eigenvalues.

Theorem 1.2 Rado [6] Let $A$ be an $n \times n$ arbitrary matrix with spectrum $\Lambda=\left\{\lambda_{1}, \lambda_{2}, \ldots, \lambda_{n}\right\}$. Let $X=\left[\mathbf{x}_{1}|\cdots| \mathbf{x}_{r}\right]$ be such that $\operatorname{rank}(X)=r$ and $A \mathbf{x}_{i}=\lambda_{i} \mathbf{x}_{i}, i=1, \ldots, r, r \leq n$. Let $C$ be an $r \times n$ arbitrary matrix. Then $A+$ $X C$ has eigenvalues $\mu_{1}, \ldots, \mu_{r}, \lambda_{r+1}, \ldots \lambda_{n}$, where $\mu_{1}, \ldots, \mu_{r}$ are eigenvalues of the matrix $\Omega+C X$ with $\Omega=\operatorname{diag}\left\{\lambda_{1}, \ldots, \lambda_{r}\right\}$.

The case $r=1$ in Theorem 1.2, constitutes a well known theorem of Brauer [1, Theorem 27], also exploited with success in connection with the NIEP. In [9] the authors introduce a symmetric version of Rado's Theorem 1.2, that is, if $A$ and $C$ are symmetric, and $\mathbf{x}_{1}, \mathbf{x}_{2}, \ldots, \mathbf{x}_{r}$ are orthonormal eigenvectors of $A$, then $A+X C X^{T}$ is also symmetric with eigenvalues $\mu_{1}, \ldots, \mu_{r}, \lambda_{r+1}, \ldots, \lambda_{n}$, where $\mu_{1}, \ldots, \mu_{r}$ are eigenvalues of the matrix $\Omega+C$. To applied this symmetric version, we need to guarantee the existence of an $r \times r$ symmetric nonnegative matrix $B=\Omega+C$ with prescribed eigenvalues and diagonal entries. This is a difficult open problem, for which some conditions are known [2]. In section 3 we give an independent sufficient condition for $n=4$.

In [3] Guo shows that if a list of complex numbers $\Lambda=\left\{\lambda_{1}, \lambda_{2}, \ldots, \lambda_{n}\right\}$, with $\lambda_{1} \geq\left|\lambda_{i}\right|, i=2, \ldots, n$, and $\lambda_{2}$ being real, is realizable, then the perturbed list 
$\Lambda_{\epsilon}=\left\{\lambda_{1}+\epsilon, \lambda_{2} \pm \epsilon, \lambda_{3}, \ldots, \lambda_{n}\right\}$ is also realizable. Guo set the question: if $\Lambda$ is symmetrically realizable, is $\Lambda_{\epsilon}$ symmetrically realizable? This is still an open question, which we consider in section 4 . We shall need the following result due to Fiedler:

Lemma 1.1 Fiedler If the lists of real numbers $\left\{\alpha_{1}, \alpha_{2}, \ldots, \alpha_{m}\right\}$ and $\left\{\beta_{1}, \beta_{2}, \ldots, \beta_{n}\right\}$ are symmetrically realizable and $\alpha_{1} \geq \beta_{1}$, then for any $\delta \geq 0$,

$$
\Lambda=\left\{\alpha_{1}+\delta, \beta_{1}-\delta, \alpha_{2}, \ldots, \alpha_{m}, \beta_{2}, \ldots, \beta_{n}\right\}
$$

is also symmetrically realizable.

We observe that Lemma 1.1 is a particular case $(r=2)$ of the Rado result.

The paper is organized as follows: In section 2 we give a sufficient condition for the existence of a symmetric nonnegative matrix with prescribed spectrum. This condition generates an algorithmic procedure to compute a solution matrix. In section 3, we introduce a sufficient condition for the existence of a $4 \times 4$ symmetric nonnegative matrix with prescribed spectrum and diagonal entries. This condition is independent of the Fiedler's sufficient condition given in $[2$, Theorem 4.4]. In section 4 we discuss about an open question of Guo [3] for symmetric nonnegative matrices and we give some partial answers. We also show some exemples to illustrate the results.

\section{A sufficient condition for SNIEP}

Let $\Lambda=\left\{\lambda_{1}, \lambda_{2}, \ldots, \lambda_{n}\right\}$ be a list of real numbers with $\lambda_{i} \geq \lambda_{i+1}, i=1, \ldots, n-1$ and $\lambda_{1} \geq\left|\lambda_{i}\right|, i=2, \ldots, n$. We shall say that $\Lambda$ is symmetrically realizable, if there exists a symmetric nonnegative matrix $A$ with spectrum $\Lambda$. The origin of this section is the following result, due to Horn [4]:

Theorem 2.1 Horn [4] Let $\left\{\mu_{i}\right\}_{i=1}^{n}$ and $\left\{\lambda_{i}\right\}_{i=1}^{n+1}$ be two sequences of real numbers such that $\lambda_{1} \geq \mu_{1} \geq \lambda_{2} \geq \mu_{2} \geq \cdots \geq \mu_{n} \geq \lambda_{n+1}$. Let

$D=\operatorname{diag}\left\{\mu_{1}, \mu_{2}, \ldots, \mu_{n}\right\}$. Then there exists a real number a and a vector $\mathbf{y} \in \mathbb{R}^{n}$ such that $\left\{\lambda_{1}, \lambda_{2}, \ldots, \lambda_{n+1}\right\}$ is the spectrum of the symmetric matrix

$$
A=\left[\begin{array}{cc}
D & \mathbf{y} \\
\mathbf{y}^{T} & a
\end{array}\right] \text {. }
$$

Next, we give the following sufficient condition, for the existence and construction of a symmetric nonnegative matrix with prescribed spectrum. 
Theorem 2.2 Let the lists of real numbers $\Lambda=\left\{\lambda_{1}, \lambda_{2}, \ldots, \lambda_{n+1}\right\}$ and $\Gamma=\left\{\mu_{1}, \mu_{2}, \ldots, \mu_{n}\right\}$ be satisfying

$$
\lambda_{1} \geq \mu_{1} \geq \lambda_{2} \geq \mu_{2} \geq \cdots \geq \mu_{n} \geq \lambda_{n+1}
$$

with $\sum_{i=1}^{n+1} \lambda_{i}-\sum_{i=1}^{n} \mu_{i} \geq 0$. Let $P$ be an orthogonal matrix and $D=\operatorname{diag}\left\{\mu_{1}, \mu_{2}, \ldots, \mu_{n}\right\}$. If $\Gamma$ is symmetrically realizable by the nonnegative matrix $B=P D P^{T}$, and $\mathbf{y}=\left(y_{1}, y_{2}, \ldots, y_{n}\right)^{T} \in \mathbb{R}^{n}$ is such that $P \mathbf{y} \geq 0$, where $y_{i}^{2}=-\frac{\Pi_{j}\left(\mu_{i}-\lambda_{j}\right)}{\Pi_{j, j \neq i}\left(\mu_{i}-\mu_{j}\right)}$, then $\Lambda$ is symmetrically realizable.

Proof. Let $a=\sum_{i=1}^{n+1} \lambda_{i}-\sum_{i=1}^{n} \mu_{i} \geq 0$.

Consider the orthogonal matrix

$$
Q=\left[\begin{array}{ll}
P & 0 \\
0 & 1
\end{array}\right]
$$

From Theorem 2.1, the matrix

$$
\bar{A}=\left[\begin{array}{cc}
D & \mathbf{y} \\
\mathbf{y}^{T} & a
\end{array}\right]
$$

has the spectrum $\Lambda=\left\{\lambda_{1}, \lambda_{2}, \ldots, \lambda_{n+1}\right\}$. Since $B$ is nonnegative and $P \mathbf{y} \geq 0$, it follows that the matrix

$$
A=\left[\begin{array}{ll}
P & 0 \\
0 & 1
\end{array}\right]\left[\begin{array}{ll}
D_{\mu} & \mathbf{y} \\
\mathbf{y}^{T} & a
\end{array}\right]\left[\begin{array}{cc}
P^{T} & 0 \\
0 & 1
\end{array}\right]=\left[\begin{array}{cc}
B & P \mathbf{y} \\
(P \mathbf{y})^{T} & a
\end{array}\right]
$$

is nonnegative with spectrum $\Lambda$.

Although the above result is not easy to apply, it allow us to decide about the realizability of certain lists, for which other criteria give no realizability information. The following examples show the usefulness of the sufficient condition given by Theorem 2.2

Example 2.1 Consider the lists $\Lambda=\{6,1,1,-4,-4\}$. According to the Spector condition [12], $\Lambda$ is symmetrically realizable. According to Lemma 1 in [10], $\Lambda$ is also the spectrum of a $5 \times 5$ nonnegative symmetric circulant matrix, but as far as we know, no other criterion in the literature about the problem allow us to decide about the symmetric realizability of this list. To construct a realizing matrix we take the auxiliary list $\Gamma=\{4,1,-1,-4\}$, interlacing the list $\Lambda$. Then $a=0 . \Gamma$ is realized by

$$
B=P \operatorname{diag}\{4,1,-1,-4\} P^{T}=\left[\begin{array}{cccc}
0 & 3 & 0 & 0 \\
3 & 0 & 0 & \frac{2}{3} \sqrt{14} \\
0 & 0 & 0 & \frac{4}{3} \\
0 & \frac{2}{3} \sqrt{14} & \frac{4}{3} & 0
\end{array}\right]
$$


where

$$
P=\left[\begin{array}{cccc}
\frac{2}{15} \sqrt{15} & -\frac{1}{30} \sqrt{210} & -\frac{1}{30} \sqrt{210} & \frac{2}{15} \sqrt{15} \\
\frac{8}{45} \sqrt{15} & -\frac{1}{90} \sqrt{210} & \frac{1}{90} \sqrt{210} & -\frac{8}{45} \sqrt{15} \\
\frac{1}{90} \sqrt{210} & \frac{8}{45} \sqrt{15} & -\frac{8}{45} \sqrt{15} & -\frac{1}{90} \sqrt{210} \\
\frac{1}{30} \sqrt{210} & \frac{2}{15} \sqrt{15} & \frac{2}{15} \sqrt{15} & \frac{1}{30} \sqrt{210}
\end{array}\right]
$$

We compute $\mathbf{y}=\left(\sqrt{\frac{48}{5}}, 0,-\sqrt{\frac{42}{5}}, 0\right)^{T}$. Then the matrix

$$
\begin{aligned}
A & =\left[\begin{array}{ll}
P & 0 \\
0 & 1
\end{array}\right]\left[\begin{array}{cccc}
\operatorname{diag}\{4,1,-1,4\} & y \\
y^{T} & & 0
\end{array}\right]\left[\begin{array}{ccc}
P^{T} & 0 \\
0 & 1
\end{array}\right] \\
& =\left[\begin{array}{cccccc}
0 & 3 & 0 & 0 & 3 \\
3 & 0 & 0 & \frac{2}{3} \sqrt{14} & \frac{5}{3} \\
0 & 0 & 0 & \frac{4}{3} & \frac{2}{3} \sqrt{14} \\
0 & \frac{2}{3} \sqrt{14} & \frac{4}{3} & 0 & 0 \\
3 & \frac{5}{3} & \frac{2}{3} \sqrt{14} & 0 & 0
\end{array}\right]
\end{aligned}
$$

is symmetric nonnegative with spectrum $\Lambda$.

Example 2.2 Let us consider the list $\Lambda=\{9,8,-3,-5,-7\}$. The Spector necessary condition iii), in Theorem 1.1, is not satisfied for the shifted list $\Lambda-\frac{2}{5}$ (zero trace). We show that $\Lambda$ is the spectrum of a symmetric nonnegative matrix: Let $\Gamma=\{9,3,-3,-7\}$. Then $D=\operatorname{diag}\{9,3,-3,-7\}, a=0, \mathbf{y}=$ $(0, \sqrt{40}, 0,0)^{T}$,

$$
P=\left[\begin{array}{cccc}
0 & \frac{1}{\sqrt{2}} & -\frac{1}{\sqrt{2}} & 0 \\
0 & \frac{1}{\sqrt{2}} & \frac{1}{\sqrt{2}} & 0 \\
\frac{1}{\sqrt{2}} & 0 & 0 & -\frac{1}{\sqrt{2}} \\
\frac{1}{\sqrt{2}} & 0 & 0 & \frac{1}{\sqrt{2}}
\end{array}\right], \quad P \mathbf{y} \geq 0
$$

and

$$
B=P D P^{T}=\left[\begin{array}{llll}
0 & 3 & 0 & 0 \\
3 & 0 & 0 & 0 \\
0 & 0 & 1 & 8 \\
0 & 0 & 8 & 1
\end{array}\right]
$$

Thus,

$$
A=\left[\begin{array}{ccccc}
0 & 3 & 0 & 0 & \sqrt{20} \\
3 & 0 & 0 & 0 & \sqrt{20} \\
0 & 0 & 1 & 8 & 0 \\
0 & 0 & 8 & 1 & 0 \\
\sqrt{20} & \sqrt{20} & 0 & 0 & 0
\end{array}\right]
$$

is symmetric nonnegative with spectrum $\Lambda$. 
The procedure in the above examples requires to find a $4 \times 4$ orthogonal matrix $P$ such that $P D P^{T}$ is nonnegative whith $D=\operatorname{diag}\left\{\mu_{1}, \ldots, \mu_{4}\right\}$, and $P \mathbf{y} \geq 0$. Then we have

Corollary 2.1 Let $\Lambda=\left\{\lambda_{1}, \lambda_{2}, \lambda_{3}, \lambda_{4}, \lambda_{5}\right\}, \Gamma=\left\{\mu_{1}, \mu_{2}, \mu_{3}, \mu_{4}\right\}$ be such that $\lambda_{i} \geq \mu_{i} \geq \lambda_{i+1}, i=1, \ldots, 4, \Sigma_{i=1}^{5} \lambda_{i}-\Sigma_{i=1}^{4} \mu_{i} \geq 0, \mathbf{y} \in \mathbb{R}^{4}$. If any of the following statement is true:

1. $P_{1} \mathbf{y} \geq 0 \wedge \mu_{3} \geq 0>\mu_{4}$,

2. $P_{2} \mathbf{y} \geq 0 \wedge \mu_{2} \geq 0>\mu_{3} \wedge \mu_{2} \geq\left|\mu_{3}\right|$,

3. $P_{3} \mathbf{y} \geq 0 \wedge \mu_{2} \geq 0>\mu_{3} \wedge \mu_{2}<\left|\mu_{3}\right|$, where

4. $P_{4} \mathbf{y} \geq 0 \wedge \mu_{1} \geq 0>\mu_{2}$

$$
\begin{aligned}
& P_{1}=\left[\begin{array}{cccc}
\frac{1}{\sqrt{2}} & 0 & 0 & -\frac{1}{\sqrt{2}} \\
\frac{1}{\sqrt{2}} & 0 & 0 & \frac{1}{\sqrt{2}} \\
0 & 1 & 0 & 0 \\
0 & 0 & 1 & 0
\end{array}\right], P_{2}=\left[\begin{array}{cccc}
0 & \frac{1}{\sqrt{2}} & -\frac{1}{\sqrt{2}} & 0 \\
0 & \frac{1}{\sqrt{2}} & \frac{1}{\sqrt{2}} & 0 \\
\frac{1}{\sqrt{2}} & 0 & 0 & -\frac{1}{\sqrt{2}} \\
\frac{1}{\sqrt{2}} & 0 & 0 & \frac{1}{\sqrt{2}}
\end{array}\right] \\
& P_{3}=\left[\begin{array}{cccc}
\frac{\sqrt{\mu_{1}+\mu_{3}}}{\sqrt{2} \sqrt{\mu_{1}-\mu_{2}}} & -\frac{\sqrt{-\left(\mu_{2}+\mu_{3}\right)}}{\sqrt{2} \sqrt{\mu_{1}-\mu_{2}}} & 0 & -\frac{1}{\sqrt{2}} \\
\frac{\sqrt{\mu_{1}+\mu_{3}}}{\sqrt{2} \sqrt{\mu_{1}-\mu_{2}}} & -\frac{\sqrt{-\left(\mu_{2}+\mu_{3}\right)}}{\sqrt{2} \sqrt{\mu_{1}-\mu_{2}}} & 0 & \frac{1}{\sqrt{2}} \\
\frac{\sqrt{-\left(\mu_{2}+\mu_{3}\right)}}{\sqrt{2} \sqrt{\mu_{1}-\mu_{2}}} & \frac{\sqrt{\mu_{1}+\mu_{3}}}{\sqrt{2} \sqrt{\mu_{1}-\mu_{2}}} & -\frac{1}{\sqrt{2}} & 0 \\
\frac{\sqrt{-\left(\mu_{2}+\mu_{3}\right)}}{\sqrt{2} \sqrt{\mu_{1}-\mu_{2}}} & \frac{\sqrt{\mu_{1}+\mu_{3}}}{\sqrt{2} \sqrt{\mu_{1}-\mu_{2}}} & \frac{1}{\sqrt{2}} & 0
\end{array}\right] \\
& P_{4}=\left[\begin{array}{cccc}
\frac{\sqrt{\mu_{1}} \sqrt{\mu_{1}+\mu_{2}}}{\sqrt{2} \sqrt{\mu_{1}+\mu_{2}-\mu_{3}} \sqrt{\mu_{1}-\mu_{2}}} & -\frac{\sqrt{-\mu_{2}} \sqrt{\mu_{1}+\mu_{2}}}{\sqrt{2} \sqrt{\mu_{1}+\mu_{2}-\mu_{3}} \sqrt{\mu_{1}-\mu_{2}}} & -\frac{\sqrt{-\mu_{3}}}{\sqrt{2} \sqrt{\mu_{1}+\mu_{2}}-\mu_{3}} & -\frac{1}{\sqrt{2}} \\
\frac{\sqrt{\mu_{1}} \sqrt{\mu_{1}+\mu_{2}}}{\sqrt{2} \sqrt{\mu_{1}+\mu_{2}-\mu_{3}} \sqrt{\mu_{1}-\mu_{2}}} & -\frac{\sqrt{-\mu_{2}} \sqrt{\mu_{1}+\mu_{2}}}{\sqrt{2} \sqrt{\mu_{1}+\mu_{2}-\mu_{3}} \sqrt{\mu_{1}-\mu_{2}}} & -\frac{\sqrt{-\mu_{3}}}{\sqrt{2} \sqrt{\mu_{1}+\mu_{2}-\mu_{3}}} & \frac{1}{\sqrt{2}} \\
\frac{\sqrt{\mu_{1}} \sqrt{-\mu_{3}}}{\sqrt{\mu_{1}+\mu_{2}-\mu_{3}} \sqrt{\mu_{1}-\mu_{2}}} & -\frac{\sqrt{-\mu_{2}} \sqrt{-\mu_{3}}}{\sqrt{\mu_{1}+\mu_{2}-\mu_{3}} \sqrt{\mu_{1}-\mu_{2}}} & \frac{\sqrt{\mu_{1}+\mu_{2}}}{\sqrt{\mu_{1}+\mu_{2}-\mu_{3}}} & 0 \\
\frac{\sqrt{-\mu_{2}}}{\sqrt{\mu_{1}-\mu_{2}}} & \frac{\sqrt{\mu_{1}}}{\sqrt{\mu_{1}-\mu_{2}}} & 0 & 0
\end{array}\right],
\end{aligned}
$$

then $\Lambda$ is symmetrically realizable.

Proof. We recall that $\mathbf{y} \in \mathbb{R}^{4}$ is defined by $y_{i}^{2}=-\frac{\Pi_{j}\left(\mu_{i}-\lambda_{j}\right)}{\Pi_{j, j \neq i}\left(\mu_{i}-\mu_{j}\right)}$. We shall consider only the case 1 . The proofs for the other cases are similar 1. $P_{1} \mathbf{y} \geq 0$ and $\mu_{3} \geq 0>\mu_{4}$. Then we define the symmetric matrix

$$
A=\left[\begin{array}{ccccc}
\frac{1}{\sqrt{2}} & 0 & 0 & -\frac{1}{\sqrt{2}} & 0 \\
\frac{1}{\sqrt{2}} & 0 & 0 & \frac{1}{\sqrt{2}} & 0 \\
0 & 1 & 0 & 0 & 0 \\
0 & 0 & 1 & 0 & 0 \\
0 & 0 & 0 & 0 & 1
\end{array}\right]\left[\begin{array}{ccccc}
\mu_{1} & 0 & 0 & 0 & y_{1} \\
0 & \mu_{2} & 0 & 0 & y_{2} \\
0 & 0 & \mu_{3} & 0 & y_{3} \\
0 & 0 & 0 & \mu_{4} & y_{4} \\
y_{1} & y_{2} & y_{3} & y_{4} & a
\end{array}\right]\left[\begin{array}{ccccc}
\frac{1}{\sqrt{2}} & \frac{1}{\sqrt{2}} & 0 & 0 & 0 \\
0 & 0 & 1 & 0 & 0 \\
0 & 0 & 0 & 1 & 0 \\
-\frac{1}{\sqrt{2}} & \frac{1}{\sqrt{2}} & 0 & 0 & 0 \\
0 & 0 & 0 & 0 & 1
\end{array}\right]
$$




$$
A=\left[\begin{array}{ccccc}
\frac{1}{2} \mu_{1}+\frac{1}{2} \mu_{4} & \frac{1}{2} \mu_{1}-\frac{1}{2} \mu_{4} & 0 & 0 & \frac{1}{2} \sqrt{2} y_{1}-\frac{1}{2} \sqrt{2} y_{4} \\
\frac{1}{2} \mu_{1}-\frac{1}{2} \mu_{4} & \frac{1}{2} \mu_{1}+\frac{1}{2} \mu_{4} & 0 & 0 & \frac{1}{2} \sqrt{2} y_{1}+\frac{1}{2} \sqrt{2} y_{4} \\
0 & 0 & \mu_{2} & 0 & y_{2} \\
0 & 0 & 0 & \mu_{3} & y_{3} \\
\frac{1}{2} \sqrt{2} y_{1}-\frac{1}{2} \sqrt{2} y_{4} & \frac{1}{2} \sqrt{2} y_{1}+\frac{1}{2} \sqrt{2} y_{4} & y_{2} & y_{3} & a
\end{array}\right]
$$

It is clear that $\mu_{1} \geq\left|\mu_{i}\right|, i=2,3,4$. Thus $\frac{1}{2} \mu_{1} \pm \frac{1}{2} \mu_{4} \geq 0$. As $\mu_{3} \geq 0$, then $\mu_{2} \geq 0$. Finally, since $\left(P_{1} \mathbf{y}\right)^{T} \geq 0, A$ is symmetric nonnegative with spectrum $\Lambda$.

\section{Symmetric matrices with prescribed spec- trum and diagonal entries}

A perturbation result, due to Rado, Theorem 1.2, has been very useful for both problems, the NIEP and the SNIEP. To apply this result, we need conditions for the existence of an $r \times r, r<n$, matrix with prescribed eigenvalues and diagonal entries. This is the problem we consider in this section: Given two lists of real numbers $\Lambda=\left\{\lambda_{1}, \lambda_{2}, \ldots, \lambda_{n}\right\}$, and $D=\left\{d_{1}, d_{2}, \ldots, d_{n}\right\}$, satisfying $\lambda_{1} \geq\left|\lambda_{i}\right|, i=2, \ldots, n, \lambda_{1} \geq \lambda_{2} \geq \cdots \geq \lambda_{n}$, and $d_{1} \geq d_{2} \geq \cdots \geq d_{n} \geq 0$, respectively, find sufficient conditions for the existence of a symmetric nonnegative matrix $A$ with spectrum $\Lambda$ and diagonal entries $d_{i} \in D$. Since we are given the diagonal entries $d_{1}, d_{2}, \ldots, d_{n}$, and we look for a symmetric nonnegative matrix $A$ with spectrum $\Lambda$, this problem is also a completion problem. This is a difficult open problem, for which necessary and sufficient conditions are known only for the cases $n=2$ and $n=3[2]$. For $n \geq 4$ Fiedler gave the following sufficient condition [2]:

Theorem 3.1 [2] Let $\lambda_{1} \geq \lambda_{2} \geq \cdots \geq \lambda_{n}$ and $d_{1} \geq d_{2} \geq \cdots \geq d_{n} \geq 0$ be given. If

$$
\begin{aligned}
& \sum_{i=1}^{t} \lambda_{i} \geq \sum_{i=1}^{t} d_{i}, t=1,2, \ldots, n-1, \\
& \sum_{i=1}^{n} \lambda_{i}=\sum_{i=1}^{n} d_{i} \\
& \lambda_{k} \leq d_{k-1}, k=2, \ldots, n-1,
\end{aligned}
$$

then there exists a symmetric nonnegative matrix with spectrum $\left\{\lambda_{1}, \lambda_{2}, \ldots, \lambda_{n}\right\}$ and diagonal entries $d_{1}, d_{2}, \ldots, d_{n}$.

Next we give the following sufficient condition for the case $n=4$. Since we consider the case $\lambda_{2} \geq d_{1}$, our condition is independent from the condition of Theorem 3.1. In orden to simplify the statement of this result let $\lambda_{1} \geq \lambda_{2} \geq$ $\lambda_{3} \geq \lambda_{4}$ and $d_{1} \geq d_{2} \geq d_{3} \geq d_{4}$ be lists of real numbers, with $\sum_{i=1}^{4} \lambda_{i}=$ 
$\sum_{i=1}^{4} d_{i}$, and $\lambda_{2} \geq d_{1} \geq \lambda_{3}$. Then we define

$$
\begin{aligned}
b & =\left(\lambda_{1}-d_{1}\right)\left(\lambda_{2}-d_{1}\right)\left(d_{1}-\lambda_{3}\right)\left(d_{1}-\lambda_{4}\right) \\
c & =\left(d_{1}-d_{3}\right)^{2}\left(d_{1}-d_{2}\right)^{2}+4 b \\
\mu_{1} & =\frac{d_{2}+d_{3}}{2}+\frac{1}{2} \sqrt{2 \sqrt{c}+\left(d_{1}-d_{2}\right)^{2}+\left(d_{1}-d_{3}\right)^{2}} \\
\mu_{2} & =d_{1} \\
\mu_{3} & =\frac{d_{2}+d_{3}}{2}-\frac{1}{2} \sqrt{2 \sqrt{c}+\left(d_{1}-d_{2}\right)^{2}+\left(d_{1}-d_{3}\right)^{2}},
\end{aligned}
$$

and

$$
\begin{aligned}
a & =\sqrt{\left(\mu_{1}-d_{2}\right)\left(\mu_{1}-d_{3}\right)} \\
y_{1}^{2} & =-\frac{\left(\mu_{1}-\lambda_{1}\right)\left(\mu_{1}-\lambda_{2}\right)\left(\mu_{1}-\lambda_{3}\right)\left(\mu_{1}-\lambda_{4}\right)}{\left(\mu_{1}-\mu_{2}\right)\left(\mu_{1}-\mu_{3}\right)} \\
y_{3}^{2} & =-\frac{\left(\mu_{3}-\lambda_{1}\right)\left(\mu_{3}-\lambda_{2}\right)\left(\mu_{3}-\lambda_{3}\right)\left(\mu_{3}-\lambda_{4}\right)}{\left(\mu_{3}-\mu_{1}\right)\left(\mu_{3}-\mu_{2}\right)} \\
m & =4 a^{2}+\left(d_{2}-d_{3}\right)^{2} .
\end{aligned}
$$

Theorem 3.2 Let $\lambda_{1} \geq \lambda_{2} \geq \lambda_{3} \geq \lambda_{4}$ and $d_{1} \geq d_{2} \geq d_{3} \geq d_{4}$ be lists of real numbers with $\sum_{i=1}^{4} \lambda_{i}=\sum_{i=1}^{4} d_{i}$, and $\lambda_{2} \geq d_{1} \geq \lambda_{3}$. If the inequalities

$$
\begin{gathered}
\left(\lambda_{1}-d_{2}\right)\left(\lambda_{1}-d_{3}\right)^{2}-\left(\lambda_{1}-d_{3}\right)\left(d_{1}-d_{2}\right)\left(d_{1}-d_{3}\right) \geq \\
\left(\lambda_{2}-d_{1}\right)\left(d_{1}-\lambda_{3}\right)\left(d_{1}-\lambda_{4}\right)
\end{gathered}
$$

ii)

$$
\left(\lambda_{1}-d_{1}\right)\left(\lambda_{2}-d_{1}\right)\left(d_{1}-\lambda_{3}\right)\left(d_{1}-\lambda_{4}\right) \geq
$$

$\left(\lambda_{2}-d_{2}\right)\left(\lambda_{2}-d_{3}\right)\left(\left(\lambda_{2}-d_{2}\right)\left(\lambda_{2}-d_{3}\right)-\left(d_{1}-d_{2}\right)\left(d_{1}-d_{3}\right)\right)$

iii)

$$
\left(\lambda_{1}-d_{1}\right)\left(\lambda_{2}-d_{1}\right)\left(d_{1}-\lambda_{3}\right)\left(d_{1}-\lambda_{4}\right) \geq
$$

$\left(d_{3}-\lambda_{3}\right)\left(d_{2}-\lambda_{3}\right)\left(\left(d_{3}-\lambda_{3}\right)\left(d_{2}-\lambda_{3}\right)-\left(d_{1}-d_{2}\right)\left(d_{1}-d_{3}\right)\right)$

iv) $\left(d_{3}-\lambda_{4}\right)^{2}\left(d_{2}-\lambda_{4}\right)^{2}-\left(d_{1}-d_{2}\right)\left(d_{1}-d_{3}\right)\left(d_{3}-\lambda_{4}\right)\left(d_{2}-\lambda_{4}\right) \geq$

$$
\left(\lambda_{1}-d_{1}\right)\left(\lambda_{2}-d_{1}\right)\left(d_{1}-\lambda_{3}\right)\left(d_{1}-\lambda_{4}\right)
$$

v)

$$
\left\|\mathbf{v}_{3}\right\|\left(d_{2}-d_{3}+\sqrt{m}\right) y_{1} \geq\left\|\mathbf{v}_{1}\right\|\left(d_{3}-d_{2}+\sqrt{m}\right) y_{3}
$$

where

$$
\mathbf{v}_{1}=\left[\begin{array}{c}
0 \\
\frac{d_{2}-d_{3}+\sqrt{m}}{2 a} \\
1
\end{array}\right], \mathbf{v}_{2}=\left[\begin{array}{l}
1 \\
0 \\
0
\end{array}\right], \mathbf{v}_{3}=\left[\begin{array}{c}
0 \\
\frac{d_{2}-d_{3}-\sqrt{m}}{2 a} \\
1
\end{array}\right]
$$

are satisfied, then there exists a symmetric nonnegative matrix with eigenvalues $\lambda_{1}, \lambda_{2}, \lambda_{3}, \lambda_{4}$ and diagonal entries $d_{1}, d_{2}, d_{3}, d_{4}$. 
Proof. Let

$$
B=\left[\begin{array}{ccc}
d_{1} & 0 & 0 \\
0 & d_{2} & a \\
0 & a & d_{3}
\end{array}\right]
$$

be a matrix with eigenvalues $\mu_{1}, \mu_{2}, \mu_{3}$. Then $d_{1}=\mu_{2}$ and $a=\sqrt{\left(\mu_{1}-d_{2}\right)\left(\mu_{1}-d_{3}\right)}$. Now we show that the $\mu_{i}^{\prime} s$ interlace the $\lambda_{i}^{\prime} s$. From inequality $i)$ we have

$$
\begin{aligned}
& 4\left(\lambda_{1}-d_{1}\right)\left(\lambda_{2}-d_{1}\right)\left(d_{1}-\lambda_{3}\right)\left(d_{1}-\lambda_{4}\right) \geq \\
& 4\left(\lambda_{1}-d_{1}\right)\left(\lambda_{2}-d_{1}\right)\left(d_{1}-\lambda_{3}\right)\left(d_{1}-\lambda_{4}\right)
\end{aligned}
$$

that is,

$$
\begin{aligned}
2\left(\lambda_{1}-d_{3}\right)\left(\lambda_{1}-d_{2}\right)-\left(d_{1}-d_{3}\right)\left(d_{1}-d_{2}\right) & \geq \sqrt{c} \\
2\left(\lambda_{1}\left(\lambda_{1}-d_{3}\right)-d_{2}\left(\lambda_{1}-d_{3}\right)\right)+d_{2}\left(d_{1}-d_{3}\right)-d_{1}\left(d_{1}-d_{3}\right) & \geq \sqrt{c} \\
2\left(\lambda_{1}^{2}-\lambda_{1} d_{2}-\lambda_{1} d_{3}+d_{2} d_{3}\right)-d_{2} d_{3}+d_{1} d_{2}+d_{1} d_{3}-d_{1}^{2} & \geq \sqrt{c} \\
4 \lambda_{1}^{2}-4 \lambda_{1}\left(d_{2}+d_{3}\right)+2 d_{2} d_{3}+2 d_{1} d_{2}+2 d_{1} d_{3}-2 d_{1}^{2} & \geq 2 \sqrt{c}
\end{aligned}
$$

and

$$
\begin{aligned}
\left(2 \lambda_{1}-\left(d_{2}+d_{3}\right)\right)^{2} & \geq 2 \sqrt{c}+d_{1}^{2}-2 d_{1} d_{2}+d_{2}^{2}+d_{1}^{2}-2 d_{1} d_{3}+d_{3}^{2} \\
2 \lambda_{1}-\left(d_{2}+d_{3}\right) & \geq \sqrt{2 \sqrt{c}+\left(d_{1}-d_{2}\right)^{2}+\left(d_{1}-d_{2}\right)^{2}} \\
\lambda_{1} & \geq \frac{d_{2}+d_{3}}{2}+\frac{1}{2} \sqrt{2 \sqrt{c}+\left(d_{1}-d_{2}\right)^{2}+\left(d_{1}-d_{2}\right)^{2}} \\
\lambda_{1} & \geq \mu_{1} .
\end{aligned}
$$

In the same way, from inequality $i i)$ we have

$$
\begin{aligned}
& \sqrt{c} \geq 2\left(\lambda_{2}-d_{2}\right)\left(\lambda_{2}-d_{3}\right)-\left(d_{1}-d_{2}\right)\left(d_{1}-d_{3}\right) \\
& \sqrt{c} \geq 2\left(\lambda_{2}^{2}-\lambda_{2} d_{2}-\lambda_{2} d_{3}+d_{2} d_{3}\right)+d_{1} d_{2}+d_{1} d_{3}-d_{1}^{2}-d_{2} d
\end{aligned}
$$

and

$$
\begin{aligned}
2 \sqrt{c}+d_{1}^{2}-2 d_{1} d_{2}-2 d_{1} d_{3} & \geq 4 \lambda_{2}^{2}-4 \lambda_{2}\left(d_{2}+d_{3}\right)+2 d_{2} d_{3} \\
\sqrt{2 \sqrt{c}+\left(d_{1}-d_{2}\right)^{2}+\left(d_{1}-d_{2}\right)^{2}} & \geq 2 \lambda_{2}-\left(d_{2}+d_{3}\right) \\
\frac{d_{2}+d_{3}}{2}+\frac{1}{2} \sqrt{2 \sqrt{c}+\left(d_{1}-d_{2}\right)^{2}+\left(d_{1}-d_{2}\right)^{2}} & \geq \lambda_{2} \\
\mu_{1} & \geq \lambda_{2} .
\end{aligned}
$$

In a similar way we have from inequalities $i i i)$ and $i v$ ) that $\lambda_{3} \geq \mu_{3} \geq \lambda_{4}$, and since $d_{1}=\mu_{2}$, then 


$$
\lambda_{1} \geq \mu_{1} \geq \lambda_{2} \geq \mu_{2} \geq \lambda_{3} \geq \mu_{3} \geq \lambda_{4}
$$

Now, let

$$
P=\left[\frac{\mathbf{v}_{1}}{\left\|\mathbf{v}_{1}\right\|}\left|\mathbf{v}_{2}\right| \frac{\mathbf{v}_{3}}{\left\|\mathbf{v}_{3}\right\|}\right]
$$

where

$$
\begin{aligned}
\left\|\mathbf{v}_{1}\right\| & =\sqrt{\frac{1}{4 a^{2}}\left(d_{2}-d_{3}+\sqrt{m}\right)^{2}+1} \\
\left\|\mathbf{v}_{2}\right\| & =1 \\
\left\|\mathbf{v}_{3}\right\| & =\sqrt{\frac{1}{4 a^{2}}\left(d_{2}-d_{3}-\sqrt{m}\right)^{2}+1} .
\end{aligned}
$$

$P$ is orthogonal and $B=P D P^{T}$, where $D=\operatorname{diag}\left\{\mu_{1}, \mu_{2}, \mu_{3}\right\}$. Thus we have

$$
\begin{aligned}
A & =\left[\begin{array}{ll}
P & 0 \\
0 & 1
\end{array}\right]\left[\begin{array}{ll}
\operatorname{diag}\left(\mu_{1}, \mu_{2}, \mu_{3}\right) & \mathbf{y} \\
\mathbf{y}^{T} & d_{4}
\end{array}\right]\left[\begin{array}{ll}
P^{T} & 0 \\
0 & 1
\end{array}\right] \\
& =\left[\begin{array}{ll}
B & P \mathbf{y} \\
(P \mathbf{y})^{T} & d_{4}
\end{array}\right]
\end{aligned}
$$

Finally, from $v$ ) we may see from straightforward calculation that $P \mathbf{y} \geq \mathbf{0}$, where the entries of the vector $\mathbf{y}$ are computed as in Theorem 2.2. Thus, $A$ is a $4 \times 4$ symmetric nonnegative matrix with eigenvalues $\lambda_{1}, \lambda_{2}, \lambda_{3}, \lambda_{4}$ and diagonal entries $d_{1}, d_{2}, d_{3}, d_{4}$.

Example 3.1 Consider the lists 5, 4,0,-3 and 3,3,0,0. These lists satisfy all inequalities of Theorem 3.2. Then we may compute the symmetric nonnegative matrix

$$
A=\left[\begin{array}{cccc}
3 & 0 & 0 & \sqrt{6} \\
0 & 3 & \sqrt{6} & 0 \\
0 & \sqrt{6} & 0 & 2 \\
\sqrt{6} & 0 & 2 & 0
\end{array}\right]
$$

with the desired eigenvalues and diagonal entries. Note that, since $\lambda_{2}>d_{1}$, the Fiedler's sufficient conditions does not work here.

Example 3.2 Consider the lists 7,5,0,-4, and 4, 4, 0,0. We compute the interlacing numbers $\mu_{1}=6, \mu_{2}=4, \mu_{3}=-2$, and $a=2 \sqrt{3}$. Then we have 


$$
\begin{aligned}
P D P^{T} & =\left[\begin{array}{ccc}
0 & 1 & 0 \\
\frac{1}{2} \sqrt{3} & 0 & -\frac{1}{2} \\
\frac{1}{2} & 0 & \frac{1}{2} \sqrt{3}
\end{array}\right]\left[\begin{array}{ccc}
6 & 0 & 0 \\
0 & 4 & 0 \\
0 & 0 & -2
\end{array}\right]\left[\begin{array}{ccc}
0 & \frac{1}{2} \sqrt{3} & \frac{1}{2} \\
1 & 0 & 0 \\
0 & -\frac{1}{2} & \frac{1}{2} \sqrt{3}
\end{array}\right] \\
& =\left[\begin{array}{ccc}
4 & 0 & 0 \\
0 & 4 & 2 \sqrt{3} \\
0 & 2 \sqrt{3} & 0
\end{array}\right]=B
\end{aligned}
$$

and

$$
A=\left[\begin{array}{cccc}
4 & 0 & 0 & 2 \sqrt{2} \\
0 & 4 & 2 \sqrt{3} & \frac{1}{4} \sqrt{45}-\frac{1}{4} \sqrt{21} \\
0 & 2 \sqrt{3} & 0 & \frac{1}{4} \sqrt{63}+\frac{1}{4} \sqrt{15} \\
2 \sqrt{2} & \frac{1}{4} \sqrt{45}-\frac{1}{4} \sqrt{21} & \frac{1}{4} \sqrt{63}+\frac{1}{4} \sqrt{15} & 0
\end{array}\right]
$$

with the desired spectrum and diagonal entries.

\section{On an open question of Guo}

In this section we give some partial answers to the following question of Guo [3]: If the list $\Lambda=\left\{\lambda_{1}, \lambda_{2}, \ldots, \lambda_{n}\right\}$ is symmetrically realizable, and $\epsilon>0$, is $\Lambda_{\epsilon}=\left\{\lambda_{1}+\epsilon, \lambda_{2} \pm \epsilon, \lambda_{3}, \ldots, \lambda_{n}\right\}$ also symmetrically realizable? First we show that a list $\Lambda$, which satisfies the Spector's conditions also satisfies one of the Guo perturbations:

Proposition 4.1 Let $\Lambda=\left\{\lambda_{1}, \lambda_{2}, \ldots, \lambda_{5}\right\}$, with $\sum_{i=1}^{5} \lambda_{i}=0$, be symmetrically realizable. Then $\Lambda_{\epsilon}^{-}=\left\{\lambda_{1}+\epsilon, \lambda_{2}-\varepsilon, \lambda_{3}, \lambda_{4}, \lambda_{5}\right\}$ is also symmetrically realizable.

Proof. We have

$$
s_{1}\left(\Lambda_{\epsilon}^{-}\right)=\lambda_{1}+\epsilon+\lambda_{2}-\varepsilon+\lambda_{3}+\lambda_{4}+\lambda_{5}=0 .
$$

Since $3 \epsilon \lambda_{1}^{2}+3 \epsilon^{2} \lambda_{1}-3 \epsilon \lambda_{2}^{2}+3 \epsilon^{2} \lambda_{2} \geq 0$, then

$$
\begin{aligned}
s_{3}\left(\Lambda_{\epsilon}^{-}\right) & =\left(\lambda_{1}+\epsilon\right)^{3}+\left(\lambda_{2}-\varepsilon\right)^{3}+\lambda_{3}^{3}+\lambda_{4}^{3}+\lambda_{5}^{3} \\
& =\lambda_{1}^{3}+\lambda_{2}^{3}+\lambda_{3}^{3}+\lambda_{4}^{3}+\lambda_{5}^{3}+3 \epsilon \lambda_{1}^{2}+3 \epsilon^{2} \lambda_{1}-3 \epsilon \lambda_{2}^{2}+3 \epsilon^{2} \lambda_{2} \\
& \geq 0
\end{aligned}
$$

Finally, it is clear that $\lambda_{2}-\varepsilon+\lambda_{5} \leq 0$. Then $\Lambda_{\epsilon}^{-}$is symmetrically realizable. 
Theorem 4.1 Let $\Lambda=\left\{\lambda_{1}, \lambda_{2}, \ldots, \lambda_{n}\right\}$ be a symmetrically realizable list. Then for all $\epsilon>0$ there exists $\delta>0$ such that

$$
\Lambda_{\epsilon, \delta}=\left\{\lambda_{1}+\epsilon, \lambda_{2} \pm \epsilon \delta, \ldots, \lambda_{n}\right\}
$$

is symmetrically realizable.

Proof. Let $A=P D P^{T} \geq 0$ be a symmetric nonnegative matrix with spectrum $\Lambda$, where $P=\left[\mathbf{p}_{1}\left|\mathbf{p}_{2}\right| \cdots \mid \mathbf{p}_{n}\right]$ is an orthogonal matrix and $D=\operatorname{diag}\left\{\lambda_{1}, \lambda_{2}, \ldots, \lambda_{n}\right\}$.

Then we have

$$
A=\lambda_{1} \mathbf{p}_{1} \mathbf{p}_{1}^{T}+\lambda_{2} \mathbf{p}_{2} \mathbf{p}_{2}^{T}+\cdots+\lambda_{n} \mathbf{p}_{n} \mathbf{p}_{n}^{T}
$$

First, we consider the case $A$ reducible, i.e. $A=\oplus_{i=1}^{r} A_{i}$ is a direct sum of irreducible symmetric matrices.

Without loss of generality we assume that $\lambda_{1} \in \sigma\left(A_{1}\right), \lambda_{2} \in \sigma\left(A_{2}\right)$. Let $\mathbf{x}_{1}, \mathbf{x}_{2}$ be unitary eigenvectors of $A_{1}$ and $A_{2}$, associated to $\lambda_{1}$, and $\lambda_{2}$, respectively. Then

$$
\mathbf{p}_{1}=\left[\begin{array}{c}
\mathbf{x}_{1} \\
0
\end{array}\right], \mathbf{p}_{2}=\left[\begin{array}{c}
\mathbf{0} \\
\mathbf{x}_{2}
\end{array}\right]
$$

Thus the matrices $\mathbf{p}_{1} \mathbf{p}_{1}^{T}, \mathbf{p}_{2} \mathbf{p}_{2}^{T}$ are nonegative and

$$
\begin{aligned}
A_{\epsilon}^{+} & =\lambda_{1} \mathbf{p}_{1} \mathbf{p}_{1}^{T}+\lambda_{2} \mathbf{p}_{2} \mathbf{p}_{2}^{T}+\cdots+\lambda_{n} \mathbf{p}_{n} \mathbf{p}_{n}^{T}+\epsilon\left(\mathbf{p}_{1} \mathbf{p}_{1}^{T}+\mathbf{p}_{2} \mathbf{p}_{2}^{T}\right) \\
& =\left(\lambda_{1}+\epsilon\right) \mathbf{p}_{1} \mathbf{p}_{1}^{T}+\left(\lambda_{2}+\epsilon\right) \mathbf{p}_{2} \mathbf{p}_{2}^{T}+\ldots+\lambda_{n} \mathbf{p}_{n} \mathbf{p}_{n}^{T}
\end{aligned}
$$

is nonnegative with spectrum $\Lambda_{\epsilon}^{+}=\left\{\lambda_{1}+\epsilon, \lambda_{2}+\epsilon, \ldots, \lambda_{n}\right\}$.

On the other hand, since $\lambda_{1} \geq \lambda_{2}$, we have from Lemma 1.1 that for $\epsilon>0$,

$$
\Lambda_{\epsilon}^{-}=\left\{\lambda_{1}+\epsilon, \lambda_{2}-\epsilon, \lambda_{3}, \ldots, \lambda_{n}\right\}
$$

is symmetrically realizable. Note that for the reducible case, $\delta=1$.

Now we consider the irreducible case. Let $A=P D P^{T} \geq 0$ the symmetric matrix which realizes $\Lambda$. Then the Perron eigenvector $\mathbf{p}_{1}$ is positive. Thus, for $\mathbf{p}_{2}$, the eigenvector associated to $\lambda_{2}$, there exists $\delta>0$ such that

$$
\mathbf{p}_{1} \mathbf{p}_{1}^{T} \pm \delta \mathbf{p}_{2} \mathbf{p}_{2}^{T} \geq 0
$$

Observe that $\delta$ may be taken as $\delta=\min _{i j}\left\{\delta_{i j}:\left(\mathbf{p}_{1} \mathbf{p}_{1}^{T}\right)_{i, j} \pm \delta_{i j}\left(\mathbf{p}_{2} \mathbf{p}_{2}^{T}\right)_{i, j} \geq 0\right\}$. It follows that $\epsilon\left(\mathbf{p}_{1} \mathbf{p}_{1}^{T} \pm \delta \mathbf{p}_{2} \mathbf{p}_{2}^{T}\right) \geq 0$, for all $\epsilon>0$.

Thus, the symmetric nonnegative matrix

$$
\begin{aligned}
A_{\epsilon} & =\lambda_{1} \mathbf{p}_{1} \mathbf{p}_{1}^{T}+\lambda_{2} \mathbf{p}_{2} \mathbf{p}_{2}^{T}+\ldots+\lambda_{n} \mathbf{p}_{n} \mathbf{p}_{n}^{T}+\epsilon\left(\mathbf{p}_{1} \mathbf{p}_{1}^{T} \pm \delta \mathbf{p}_{2} \mathbf{p}_{2}^{T}\right) \\
& =\left(\lambda_{1}+\epsilon\right) \mathbf{p}_{1} \mathbf{p}_{1}^{T}+\left(\lambda_{2} \pm \delta \epsilon\right) \mathbf{p}_{2} \mathbf{p}_{2}^{T}+\lambda_{3} \mathbf{p}_{3} \mathbf{p}_{3}^{T}+\cdots+\lambda_{n} \mathbf{p}_{n} \mathbf{p}_{n}^{T}
\end{aligned}
$$

has spectrum $\left\{\lambda_{1}+\epsilon, \lambda_{2} \pm \epsilon \delta, \lambda_{3}, \ldots, \lambda_{n}\right\}$. 
Corollary 4.1 If $\Lambda=\left\{\lambda_{1}, \lambda_{2}, \ldots, \lambda_{n}\right\}$ is symmetrically realizable by a matrix $A=A_{1} \oplus A_{2}$, shuch that $\lambda_{1} \in A_{1}, \lambda_{2} \in A_{2}$, then for all $\epsilon>0$ the list $\Lambda_{\epsilon}=\left\{\lambda_{1}+\epsilon, \lambda_{2} \pm \epsilon, \ldots, \lambda_{n}\right\}$ is also symmetrically realizable.

Corollary 4.2 Let $\Lambda=\left\{\lambda_{1}, \lambda_{2}, \ldots, \lambda_{n}\right\}$ be a symmetrically realizable list. If the unitary eigenvectors $\mathbf{x}, \mathbf{y}$, associated respectively to $\lambda_{1}, \lambda_{2}$, are such that $\mathbf{x x}^{T} \pm \mathbf{y y}^{T} \geq 0$, then $\Lambda_{\epsilon}=\left\{\lambda_{1}+\epsilon, \lambda_{2} \pm \epsilon, \ldots, \lambda_{n}\right\}$ is symmetrically realizable.

Proof. Let

$$
A=\lambda_{1} \mathbf{x x}^{T}+\lambda_{2} \mathbf{y} \mathbf{y}^{T}+\lambda_{3} \mathbf{p}_{3} \mathbf{p}_{3}^{T}+\ldots+\lambda_{n} \mathbf{p}_{n} \mathbf{p}_{n}^{T} \geq 0
$$

a symmetric matrix realizing $\Lambda=\left\{\lambda_{1}, \lambda_{2}, \ldots, \lambda_{n}\right\}$. Since $\mathbf{x x}^{T} \pm \mathbf{y} \mathbf{y}^{T} \geq 0$, then from Theorem 4.1 the lists $\left\{\lambda_{1}+\epsilon, \lambda_{2} \pm \epsilon, \ldots, \lambda_{n}\right\}$ are symmetrically realizable.

In [7, Theorem 11], the author shows that $\Lambda=\left\{\lambda_{1}, \lambda_{2}, \ldots, \lambda_{n}\right\}$, with $\lambda_{1} \geq$ $\lambda_{2} \geq \cdots \geq \lambda_{n}$, is realizable by a nonnegative matrix if

$$
\lambda_{1}+\lambda_{n}+\sum_{S_{k}<0} S_{k} \geq 0
$$

where $S_{k}=\lambda_{k}+\lambda_{n-k+1}, k=2, \ldots, n$. This sufficient condition is called the $S 1$ criterion. In [8] it was shown that if $\Lambda$ satisfies condition (2), then $\Lambda$ is also realizable by a symmetric nonnegative matrix. Next we show that the Guo's question, introduced at the begining of this section, has a positive answer for this kind of realizations.

Theorem 4.2 If $\Lambda=\left\{\lambda_{1}, \lambda_{2}, \ldots, \lambda_{n}\right\}$ is S1-realizable, then

$$
\Lambda_{\epsilon}=\left\{\lambda_{1}+\epsilon, \lambda_{2} \pm \epsilon, \lambda_{3}, \ldots, \lambda_{n}\right\}
$$

is symmetrically realizable.

Proof. It is clear that $\Lambda$ is symmetrically realizable and that we may assume $\lambda_{1}+\epsilon+\lambda_{n}+\sum_{S_{k}<0} S_{k}=0$. Consider the partition of $\Lambda_{\epsilon}$ into

$$
\begin{aligned}
& \Lambda_{1}=\left\{\lambda_{1}+\epsilon, \lambda_{n}\right\}, \quad \Lambda_{2}=\left\{\lambda_{2} \pm \epsilon, \lambda_{n-1}\right\}, \\
& \Lambda_{k}=\left\{\lambda_{k}, \lambda_{n-k+1}\right\}, \quad k=3, \ldots,\left[\frac{n}{2}\right],
\end{aligned}
$$

with $\Lambda_{\frac{n+1}{2}}=\left\{\lambda_{\frac{n+1}{2}}\right\}$ for $n$ odd. From now on we shall consider $n$ as even number. The proof for odd $n$ is similar. Observe that some of the lists $\Lambda_{k}$ can be realizable, while some other are nonnrealizable. Without loss of generality we assume that $\Lambda_{2}, \Lambda_{3}, \ldots, \Lambda_{t}, t \leq\left[\frac{n}{2}\right]$, are nonrealizable $\left(S_{k}<0\right.$ for $k=$ $2, \ldots, t)$, and that $\Lambda_{t+1}, \ldots, \Lambda_{\left[\frac{n}{2}\right]}$ (if there is some one) are realizable lists 
$\left(S_{k} \geq 0\right.$ for $\left.k=t+1, \ldots,\left[\frac{n}{2}\right]\right)$ by, we say, the nonnegative symmetric matrices $B_{k}, k=t+1, \ldots,\left[\frac{n}{2}\right]$, respectively. Then $B=\oplus B_{k}$ is symmetric nonnegative with spectrum $\Lambda_{t+1} \cup \cdots \cup \Lambda_{\left[\frac{n}{2}\right]}$. Let us consider now, if there is some one, the nonnrealizable lists $\Lambda_{k}, k=2,3, \ldots, t$, together with the realizable list $\Lambda_{1}$. We renumber the $2 t$ elements in $\cup \Lambda_{k}, k=1, \ldots, t$, as

$$
\lambda_{1} \geq \lambda_{2} \geq \cdots \geq \lambda_{t} \geq \lambda_{t+1} \geq \cdots \geq \lambda_{2 t}
$$

For each one of the lists $\Lambda_{k}, k=1,2, \ldots, t$, we define the associated symmetric realizable lists

$$
\Gamma_{k}=\left\{-\lambda_{2 t-k+1}, \lambda_{2 t-k+1}\right\}
$$

We start by merging the lists

$$
\Gamma_{1}=\left\{-\lambda_{2 t}, \lambda_{2 t}\right\} \text { and } \Gamma_{2}=\left\{-\lambda_{2 t-1}, \lambda_{2 t-1}\right\} .
$$

Let $\delta_{2}=-\lambda_{2 t-1}-\lambda_{2}+\epsilon=-S_{2}$ Then $\delta_{2}=-S_{2}>0$ and from Lemma 1.1 we have that

$$
\begin{aligned}
\Omega_{2} & =\left\{-\lambda_{2 t}+\delta_{2},-\lambda_{2 t-1}-\delta_{2}, \lambda_{2 t-1}, \lambda_{2 t}\right\} \\
& =\left\{-\lambda_{2 t}-\lambda_{2 t-1}-\lambda_{2}+\epsilon, \lambda_{2}-\epsilon, \lambda_{2 t-1}, \lambda_{2 t}\right\}
\end{aligned}
$$

is symmetrically realizable by a $4 \times 4$ matrix. Next we merge $\Omega_{2}$ with $\Gamma_{3}=$ $\left\{-\lambda_{2 t-2}, \lambda_{2 t-2}\right\}$, and obtain for $\delta_{3}=-\left(\lambda_{3}+\lambda_{2 t-2}\right)=-S_{3}$,

$$
\begin{aligned}
\Omega_{3} & =\left\{-\lambda_{2 t}-\lambda_{2 t-1}-\lambda_{2}+\epsilon+\delta_{3},-\lambda_{2 t-2}-\delta_{3}, \lambda_{2}-\epsilon, \lambda_{2 t-2}, \lambda_{2 t-1}, \lambda_{2 t}\right\} \\
& =\left\{-\lambda_{2 t}-\lambda_{2 t-1}-\lambda_{2 t-2}-\lambda_{2}-\lambda_{3}+\epsilon, \lambda_{3}, \lambda_{2}-\epsilon, \lambda_{2 t-2}, \lambda_{2 t-1}, \lambda_{2 t}\right\},
\end{aligned}
$$

which is symmetrically realizable by a $6 \times 6$ matrix. We continue this procedure until in the last step we merge

$$
\Omega_{t-1}=\left\{-\lambda_{2 t}-\sum_{k=2}^{t-1} S_{k}+\epsilon, \lambda_{t-1}, *, \ldots, *\right\} \text { with } \Gamma_{t}=\left\{-\lambda_{t+1}, \lambda_{t+1}\right\}
$$

to obtain, with $\delta_{t}=S_{t}=-\left(\lambda_{t}+\lambda_{t+1}\right)$,

$$
\begin{gathered}
\Lambda_{\epsilon}^{-}=\left\{-\lambda_{2 t}-\sum_{k=2}^{t} S_{k}+\epsilon, \lambda_{t}, *, \ldots, *\right\} \\
\Lambda_{\epsilon}^{-}=\left\{-\lambda_{n}-\sum_{S_{k}<0} S_{k}+\epsilon, \lambda_{t}, *, \ldots, *\right\} \\
\left\{\lambda_{1}+\epsilon, \lambda_{2}-\epsilon, \lambda_{3}, \ldots, \lambda_{n}\right\},
\end{gathered}
$$


which is symmetrically realizable.

For the list $\Lambda_{\epsilon}^{+}=\left\{\lambda_{1}+\epsilon, \lambda_{2}+\epsilon, \lambda_{3}, \ldots, \lambda_{n}\right\}$ we observe that if $\lambda_{2}+\lambda_{n-1} \geq 0$, then $S_{2}=\lambda_{2}+\epsilon+\lambda_{n-1} \geq 0$ and

$$
\lambda_{1}+\epsilon+\lambda_{n}+\sum_{S_{k}} S_{k} \geq 0
$$

while if $\lambda_{2}+\lambda_{n-1}<0$, then $S_{2}=\lambda_{2}+\epsilon+\lambda_{n-1} \geq 0$ and (3) is also satisfied, or $S_{2}=\lambda_{2}+\epsilon+\lambda_{n-1}<0$ with

$$
S_{2}=\lambda_{2}+\epsilon+\lambda_{n-1}>\lambda_{2}+\lambda_{n-1}
$$

and (3) is again satisfied. Hence, $\Lambda_{\epsilon}^{+}$is $S 1$-realizable and from [8, Lemma 4] it is symmetrically realizable, we say, by a nonnegative matrix $A$. Thus, $\Lambda$ symmetrically realizable by $A \oplus B$.

\section{References}

[1] A. Brauer, Limits for the characteristic roots of a matrix IV: Applications to stochastic matrices, Duke Math. J. 19 (1952) 75-91.

[2] M. Fiedler, Eigenvalues of nonnegative symmetric matrices, Linear Algebra Appl. 9 (1974) 119-142.

[3] W. Guo, Eigenvalue of Nonnegative Matrices, Linear Algebra and its Applications 266(1997) 261-270.

[4] A. Horn, Doubly stochastic matrices and the diagonal of matrix rotations, Amer. J. Math., 76(1954) 620-630.

[5] T. J. Laffey, H. Smigoc, Construction of nonnegative symmetric matrices with given spectrum, Linear Algebra Appl. 421 (2007) 97-109.

[6] H. Perfect, Methods of constructing certain stochastics matrices II, Duke Math. J. 22(1955) 305-311.

[7] R.L. Soto, Existence and construction of nonnegative matrices with prescribed spectrum, Linear Algebra Appl. 369 (2003) 169-184.

[8] R. L. Soto, Realizability criterion for the symmetric nonnegative inverse eigenvalue problem, Linear Algebra Appl. 416 (2006) 783-794.

[9] R. L. Soto, O. Rojo, J. Moro, A. Borobia, Symmetric nonnegative inverse eigenvalue problem, Electron. J. Linear Algebra 16 (2007) 1-18. 
[10] R. L. Soto, O. Rojo, Applications of a Brauer theorem in the nonnegative inverse eigenvalue problem, Linear Algebra Appl. 416 (2006) 844-856.

[11] G. Soules, Constructing Symmetric Nonnegative Matrices, Linear Multilinear Algebra 13 (1983) 241-251.

[12] O. Spector, A characterization of trace zero symmetric nonneg-ative $5 \times 5$ matrices,Linear Algebra ans its Applications, (2011) 434:1000-1017.

Received: June 11, 2014 